\title{
Radiation and cardiovascular disease
}

\author{
Nicolas Palaskas ${ }^{1}$, Ashley Patel $^{2}$, Syed Wamique Yusuf ${ }^{1}$ \\ ${ }^{1}$ University of Texas MD Anderson Cancer Center, Houston, Texas, USA; ${ }^{2}$ Baylor College of Medicine, Houston, Texas, USA \\ Correspondence to: Syed Wamique Yusuf. Department of Cardiology, University of Texas MD Anderson Cancer Center, 1515 Holcombe Blvd. Unit \\ 1451, Houston, Texas 77030, USA. Email: syusuf@mdanderson.org. \\ Provenance: This is an invited article commissioned by the Section Editor Dr. Hsin-Hua Nien, MD (Attending physician, Department of Radiation \\ Oncology, Cathay General Hospital, Taipei). \\ Comment on: Demissei BG, Freedman G, Feigenberg SJ, et al. Early Changes in Cardiovascular Biomarkers with Contemporary Thoracic Radiation \\ Therapy for Breast Cancer, Lung Cancer, and Lymphoma. Int J Radiat Oncol Biol Phys 2019;103:851-60.
}

Submitted Aug 17, 2019. Accepted for publication Aug 28, 2019.

doi: 10.21037/atm.2019.08.107

View this article at: http://dx.doi.org/10.21037/atm.2019.08.107

Cardiovascular disease and cancer are the two leading causes of death in the US (1). By year 2030, it is estimated that there will be more than 22.1 million cancer survivors in US (2). With increasing cancer survivorship, further emphasis has been placed on reducing cancer treatment related cardiotoxicity. Radiotherapy is a common cancer therapeutic strategy and is especially important in treating breast cancer, lung cancer, and lymphomas. Autopsy studies have shown that patients treated with radiation therapy develop various cardiac abnormalities including coronary artery disease, pericardial disease, valvular disease, and myocardial fibrosis that may lead to heart failure both with preserved and reduced ejection fraction (3). The Swedish Council of Technology Assessment in Health Care performed a systematic review of radiation therapy for breast cancer which included 41,204 patients and it showed strong evidence of cancer disease free survival but the benefits were abrogated by strong evidence of an increased non-cancer mortality in those receiving radiotherapy (4). There was moderate evidence that cardiovascular disease was the cause of increased non-cancer mortality (4). Patients with lymphoma treated with radiotherapy showed a $21 \%$ incidence of cardiac complications which were observed late after radiotherapy (5). Patients with lung cancer receiving radiotherapy are often inoperable and have high cancer mortality but cardiac toxicity is still seen and is independently associated with mean heart dose (6).

The recognition of radiation-induced heart disease has led to several changes in how radiotherapy is administered with a focus on limiting cardiac doses. In addition, radiation techniques, such as breath holds and computerized tomography (CT) based treatment planning, have helped to minimize cardiac radiation. For patients with breast cancer treated with older techniques higher mortality rates due to myocardial infarction were observed for those with left sided breast cancer when compared to those with rightsided disease (7). Using modern radiotherapy techniques, the risk of cardiac mortality did not increase for patients who received left-sided irradiation for breast cancer, compared with right sided irradiation, for the 10-12 years of follow up after treatment $(8,9)$. The cardiac toxicity is dependent on several factors, which include total dose, mean heart dose, fractionation schedules, and percentage of the heart receiving radiation $(10,11)$.

Radiation induced heart disease is an all-encompassing phrase for the cardiac side effects from radiotherapy. While this term is inclusive it lacks specificity. This creates difficulty for identifying mechanisms and predictors of a multi-faceted and ill-defined outcome. In addition, heart disease and cancer have several shared risk factors. While cardiovascular events are independently associated with mean heart dose, they are also independently associated with the patient's baseline cardiovascular comorbidities and risk factors (6). Furthermore, patients with cancer receive chemotherapy in addition to radiotherapy. Anthracyclines, which have wellestablished cardiotoxicity, are commonly used in treating breast cancer and lymphomas. Patients with breast cancer over-expressing HER2 also receive trastuzumab, which has known cardiotoxicity. Patients exposed to radiation therapy are likely more predisposed to develop cardiomyopathy (12). 
In a retrospective cohort study involving 14,358 cancer survivors, it was found that survivors of cancer were significantly more likely than siblings to report congestive heart failure [hazard ratio (HR) 5.9; 95\% confidence interval (CI), 3.4 to $9.6 ; \mathrm{P}<0.001$ ]. Exposure to $250 \mathrm{mg} / \mathrm{m}^{2}$ or more of anthracyclines and radiation dose of more than $1,200 \mathrm{cGy}$ to the heart increased the relative hazard of congestive heart failure by 2- to 6-fold as compared to nonirradiated survivors (12).

The interaction among the different cardiac side effects of radiation complicates the picture. For example, radiation induced aortic stenosis can lead to heart failure but it is unclear how much and what role concomitant radiation related cardiac fibrosis plays in such cases. The task of investigators and cardiologists is to tease out the role of each of these factors, some radiation-related, some due underlying risk factors and some a combination of both.

The study by Demissei et al. (13) represents one of the few prospective studies to look at cardiac biomarker changes early after radiotherapy. The study is novel in that it examines both traditional cardiac biomarkers such as high sensitivity troponin $\mathrm{T}$ (hs-cTnT) and N-terminal B-type natriuretic peptide (NT-proBNP) in addition to a vascular biomarker in placental growth factor (PIGF) and an inflammatory biomarker in growth differentiation factor 15 (GDF-15). The hope is that by identifying early cardiac damage by changes in biomarkers we will be able to predict those patients who may develop clinical radiation induced heart disease. Earlier identification may allow for better prognostication and more aggressive monitoring or intervention for this group of selected patients.

\section{Troponin}

Cardiac biomarkers including troponins and natriuretic peptides are well-established tools for detecting ischemia and cardiac damage due to clinical conditions such as heart failure and left ventricular hypertrophy (14). They have been increasingly studied for utility as measurement tools to detect subclinical radiotherapy-induced cardiac damage. The findings of Troponin $\mathrm{T}$ decrease in both breast cancer and lymphoma/lung cancer patients presented by the authors is an interesting finding that the authors suspect was from prior anthracycline administration resulting in elevated levels at the beginning of radiotherapy (13). Early studies found that troponin $\mathrm{T}$ levels did not change acutely during radiotherapy for breast cancer, suggesting that should not be used as a marker of radiotherapy damage (15). Similarly, in a prospective study of patients with thoracic malignancy, only 2 out of 25 patients (of which one had a history of hypertension and diabetes mellitus) had transient detectable Troponin I level at the end of radiation therapy (16). Studies have shown an increase in troponin $\mathrm{T}$ level early after radiotherapy but still it is not clear if these portend to the development of clinical cardiovascular events or not $(17,18)$. These and other studies of biomarkers and their association with chemotherapeutic agents, have resulted in the inclusion of troponin assay in American Society of Echocardiography guidelines for monitoring of chemotherapy related cardiac dysfunction (19). The authors defined an elevation in troponin $\mathrm{T}$ as an increase above $14 \mathrm{ng} / \mathrm{L}$ or a $30 \%$ rise (13). It is worth noting that all patients had values less than $100 \mathrm{ng} / \mathrm{L}$ both pre and post radiotherapy with most having values less than $50 \mathrm{ng} / \mathrm{L}$ (13). It is debatable what constitutes a clinically significant cutoff of troponin T with recommendations for 52 or $100 \mathrm{ng} / \mathrm{L}$ for acute myocardial infarction (20). Regardless, the role of troponin $\mathrm{T}$ or I in monitoring or diagnosis of radiationinduced heart disease is still not clear.

\section{BNP}

B-type natriuretic peptide (BNP) and the cleavage fragment, NT-proBNP are useful biomarkers to detect myocardial strain as it is released by ventricles in response to myocyte stretch and has been implicated in the regulation of cardiac remodeling (21). The authors found no significant change in NT-proBNP levels pre and post radiotherapy as defined by a $30 \%$ elevation. Unfortunately, as with troponin $\mathrm{T}$, this is another readily available cardiac biomarker that has not clearly shown to be a predictive marker of radiation induced heart disease. One study found that even at 5 years post radiation in breast cancer patients, the BNP was still normal. Although this study did correlate the change in BNP with the mean heart and ventricular dose received (22). This same correlation between BNP and heart dose received has been observed in additional studies although later than at 1 year (23). Further long-term studies are needed to assess whether these small early incremental changes in BNP or NT-proBNP correlate with long-term clinical outcomes.

\section{PIGF, GDF-15}

PIGF and GDF-15 have been studied in the context of predicting the development and progression of 
cardiovascular disease in the general population $(24,25)$. The proposed mechanism of radiation-induced cardiotoxicity is early acute inflammation of small and medium-sized vessels, with hypoxia-induced cardiomyocyte necrosis as a result of microvascular damage and interstitial fibrosis (26). PIGF contributes to angiogenesis and atherogenesis through vascular inflammation and plaque destabilization. GDF-15, as a cytokine member of the transforming growth factor $\beta$ family is expressed and secreted in response to tissue injury, inflammation, oxidative stress, hypoxia, and oncogene activation. Demissei et al. observed that only patients with lymphoma/lung cancer who had received a higher mean cardiac radiation doses as opposed to breast cancer patients developed significant increases in PIGF and GDF (13). This provides valuable insight to understanding the pathophysiology of radiation-related cardiac injury and that at least early effects are more related to the vascular biomarkers than the traditional troponin and BNP biomarkers.

\section{LVEF and strain}

Echocardiographic imaging has demonstrated detection in early changes in left ventricular ejection fraction (LVEF) and global longitudinal left ventricular strain, which may be a promising method of identifying subclinical early regional myocardial dysfunction. LVEF decrease and strain increase were found to be related to cardiac doses (27). While it has not been delineated which cardiac chambers are affected most by radiation, some studies have found a significant association with the dose to the left ventricle (28). Factors that have been considered include how much total muscle volume compared to vascularization contributes to making the cardiac tissue more sensitive to the effects of radiation. With ventricles comprising the bulk of cardiac muscle, it has been suggested that dose escalation in the ventricles is one of the critical factors leading to radiotherapy-induced cardiac damage.

The authors did not find an association between early biomarker changes and changes in LVEF, longitudinal strain, or circumferential strain (13). Very few studies have assessed the early changes in strain after radiotherapy and most are confounded by recent cardiotoxic chemotherapeutic administration. Despite this, some small studies have seen early changes in strain and strain rate after radiotherapy. In particular Erven $e t$ al. showed leftsided breast cancer patients had reduced strain and strain rate immediately following radiotherapy that persisted at
2 months (29). Similarly, left-sided breast cancer patients receiving radiotherapy showed a worsened strain at 2 months post radiotherapy as described by Lo et al. (30). Limited evidence for early changes in strain imaging after radiotherapy exists but may have a role in predicting eventual cardiovascular events.

\section{Conclusions}

With improvement in cancer therapy, survival rates continue to improve and a greater focus is needed on the toxicities of radiotherapy. Understanding whether known biomarkers of cardiac damage, inflammation or angiogenesis can identify patients at risk of cardiotoxicity may allow for insight into radioprotective drugs, tailored early intervention, monitoring and appropriate cardiac follow-up, or modification of mediastinal radiation therapies to prevent further toxicity. The role of early changes in biomarkers and advanced imaging modalities such as strain imaging need further study for their ability to predict longterm sequelae. Possibly a combined approach of biomarkers and imaging will allow us to identify those at greatest risk.

\section{Acknowledgments}

None.

\section{Footnote}

Conflicts of Interest: The authors have no conflicts of interest to declare.

Ethical Statement: The authors are accountable for all aspects of the work in ensuring that questions related to the accuracy or integrity of any part of the work are appropriately investigated and resolved.

\section{References}

1. Heron M. Deaths: Leading causes for 2016. National Vital Statistics Reports 2018;67:1-77.

2. Miller KD, Nogueira L, Mariotto AB, et al. Cancer treatment and survivorship statistics, 2019. CA Cancer J Clin 2019. [Epub ahead of print].

3. Veinot JP, Edwards WD. Pathology of radiation-induced heart disease: a surgical and autopsy study of 27 cases. Hum Pathol 1996;27:766-73.

4. Rutqvist LE, Rose C, Cavallin-Stahl E. A systematic 
overview of radiation therapy effects in breast cancer. Acta Oncol 2003;42:532-45.

5. Girinsky T, Cordova A, Rey A, et al. Thallium-201 scintigraphy is not predictive of late cardiac complications in patients with Hodgkin's disease treated with mediastinal radiation. Int J Radiat Oncol Biol Phys 2000;48:1503-6.

6. Wang K, Eblan MJ, Deal AM, et al. Cardiac Toxicity After Radiotherapy for Stage III Non-Small-Cell Lung Cancer: Pooled Analysis of Dose-Escalation Trials Delivering 70 to 90 Gy. J Clin Oncol 2017;35:1387-94.

7. Rutqvist LE, Johansson H. Mortality by laterality of the primary tumour among 55,000 breast cancer patients from the Swedish Cancer Registry. Br J Cancer 1990;61:866-8.

8. Nixon AJ, Manola J, Gelman R, et al. No long-term increase in cardiac-related mortality after breast conserving surgery and radiation therapy using modern techniques. J Clin Oncol 1998;16:1374-9.

9. Vallis KA, Pintilie M, Chong N, et al. Assessment of coronary heart disease morbidity and mortality after radiation therapy for early breast cancer. J Clin Oncol 2002;20:1036-42.

10. Gayed IW, Liu HH, Wei X et al. Patterns of cardiac perfusion abnormalities after chemoradiotherapy in patients with lung cancer. J Thorac Oncol 2009;4:179-84.

11. Gayed IW, Liu HH, Yusuf SW et al. The prevalence of myocardial ischemia after concurrent chemoradiation therapy as detected by gated myocardial perfusion imaging in patients with esophageal cancer. J Nucl Med 2006;47:1756-62.

12. Mulrooney DA, Yeazel MW, Kawashima T, et al. Cardiac outcomes in a cohort of adult survivors of childhood and adolescent cancer: retrospective analysis of the Childhood Cancer Survivor Study cohort. BMJ 2009;339:b4606.

13. Demissei BG, Freedman G, Feigenberg SJ, et al. Early Changes in Cardiovascular Biomarkers with Contemporary Thoracic Radiation Therapy for Breast Cancer, Lung Cancer, and Lymphoma. Int J Radiat Oncol Biol Phys 2019;103:851-60.

14. Chow SL, Maisel AS, Anand I, et al. Role of Biomarkers for the Prevention, Assessment, and Management of Heart Failure: A Scientific Statement From the American Heart Association. Circulation 2017;135:e1054-91.

15. Hughes-Davies L, Sacks D, Rescigno J, et al. Serum cardiac troponin $\mathrm{T}$ levels during treatment of early-stage breast cancer. J Clin Oncol 1995;13:2582-4.

16. Gomez DR, Yusuf SW, Munsell MF et al. Prospective Exploratory Analysis of Cardiac Biomarkersand Electrocardiogram Abnormalities in Patients Receiving
Thoracic Radiation Therapy with High-Dose Heart Exposure. J Thorac Oncol 2014;9:1554-60.

17. Skyttä T, Tuohinen S, Boman E, et al. Troponin T-release associates with cardiac radiation doses during adjuvant left-sided breast cancer radiotherapy. Radiat Oncol 2015;10:141.

18. Erven K, Florian A, Slagmolen P, et al. Subclinical cardiotoxicity detected by strain rate imaging up to 14 months after breast radiationtherapy. Int J Radiat Oncol Biol Phys 2013;85:1172-8.

19. Plana JC, Galderisi M, Barac A, et al. Expert consensus for multimodality imaging evaluation of adult patients during and after cancer therapy: a report from the American Society of Echocardiography and the European Association of Cardiovascular Imaging. J Am Soc Echocardiogr 2014;27:911-39.

20. Vasile V, Jaffe A. High-Sensitivity Cardiac Troponin in the Evaluation of Possible AMI. American College of Cardiology: Latest in Cardiology. American College of Cardiology Foundation. July 16, 2018. Available online: https://www.acc.org/latest-in cardiology/ articles/2018/07/16/09/17/high-sensitivity-cardiactroponin-in-the-evaluation-of-possible-ami. Accessed June 21, 2019.

21. Mant J, Doust J, Roalfe A, et al. Systematic review and individual patient data meta-analysis of diagnosis of heart failure, with modelling of implications of different diagnostic strategies in primary care. Health Technol Assess 2009;13:1-207, iii.

22. Portaluri M, Petruzzelli MF, Tramacere F, et al. B-type natriuretic peptide plasma level in 5-year breast cancer survivors after radiotherapy. Int J Radiat Biol 2019;95:201-6.

23. D'Errico MP, Petruzzelli MF, Gianicolo EA, et al. Kinetics of B-type natriuretic peptide plasma levels in patients with left-sided breast cancer treated with radiation therapy: Results after one-year follow-up. Int J Radiat Biol 2015;91:804-9.

24. Cassidy A, Chiuve SE, Manson JE, et al. Potential role for plasma placental growth factor in predicting coronary heart disease risk in women. Arterioscler Thromb Vasc Biol 2009;29:134-9.

25. Wollert KC, Kempf T, Wallentin L. Growth Differentiation Factor 15 as a Biomarker in Cardiovascular Disease. Clin Chem 2017;63:140-51.

26. Spetz J, Moslehi J, Sarosiek K. Radiation-Induced Cardiovascular Toxicity: Mechanisms, Prevention, and Treatment. Curr Treat Options Cardiovasc Med 
2018;20:31.

27. Donovan EK, Dhesy-Thind S, Swaminath A, et al. MEDiastinal Irradiation and CArdio-Toxic Effects (MEDICATE): Exploring the Relationship between Cardiac Irradiation and High Sensitivity Troponins. Clin Oncol (R Coll Radiol) 2019;31:479-85.

28. Jacob S, Camilleri J, Derreumaux S, et al. Is mean heart dose a relevant surrogate parameter of left ventricle and coronary arteries exposure during breast cancer radiotherapy: a dosimetric evaluation based on

Cite this article as: Palaskas N, Patel A, Yusuf SW. Radiation and cardiovascular disease. Ann Transl Med 2019;7(Suppl 8):S371. doi: 10.21037/atm.2019.08.107 individually-determined radiation dose (BACCARAT study). Radiat Oncol 2019;14:29.

29. Erven K, Jurcut R, Weltens C, et al. Acute radiation effects on cardiac function detected by strain rate imaging in breast cancer patients. Int J Radiat Oncol Biol Phys 2011;79:1444-51.

30. Lo Q, Hee L, Batumalai V, et al. Sub-clinical cardiac dysfunction detected by strain imaging during breast irradiation with persistent changes 6 weeks after treatment. Int J Radiat Oncol Biol Phys 2015;92:268-76. 